\title{
BMJ An assessment of the iPad as a testing Open platform for distance visual acuity in adults
}

\author{
J M Black, R J Jacobs, G Phillips, L Chen, E Tan, A Tran, B Thompson
}

To cite: Black JM, Jacobs RJ, Phillips G, et al. An assessment of the iPad as a testing platform for distance visual acuity in adults. BMJ Open 2013;3:e002730. doi:10.1136/bmjopen-2013002730

- Prepublication history for this paper is available online. To view these files please visit the journal online (http://dx.doi.org/10.1136/ bmjopen-2013-002730).

Received 6 March 2013 Revised 18 May 2013 Accepted 20 May 2013

This final article is available for use under the terms of the Creative Commons Attribution Non-Commercial 2.0 Licence; see http://bmjopen.bmj.com

Department of Optometry and Vision Science, University of Auckland, Auckland, New Zealand

Correspondence to Dr J M Black, j.black@auckland.ac.nz

\section{ABSTRACT}

Objectives: Visual acuity is a common measurement in general practice, and the advent of new technology such as tablet computers offers a change in the way in which these tests are delivered. The aim of this study was to assess whether measurements of distance visual acuity using LogMAR letter charts displayed on an iPad tablet computer were in agreement with standard clinical tests of visual acuity in adults with normal vision.

Design: Blinded, diagnostic test study.

Setting: Single centre (University) in Auckland, New Zealand.

Participants: University staff and students $(n=85)$. Participants were required to have visual acuity better than $6 / 60$ and wear habitual refractive correction during testing. Participants were excluded if there was any history of ocular pathology.

\section{Primary and secondary outcome measures:}

Visual acuity measured under a number of conditions. Results: The iPad tablet with its glossy screen was highly susceptible to glare resulting in acuity measurements that were significantly poorer (approximately 2 LogMAR lines) than those made using an ETDRS chart and a standard computerised testing system $(n=56)$. However, fitting the iPad with an antiglare screen and positioning the device away from sources creating reflected (veiling) glare resulted in acuity measurements that were equivalent those made using gold standard charts $(n=29)$.

Conclusions: Tablet computers are an attractive option for visual acuity measurement due to portability, the ability to randomise letters, automated scoring of acuity and the ability to select from a range of charts. However, these devices are only suitable for use in situations where sources of glare can be eliminated.

\section{INTRODUCTION}

Recent advances in technology represent a valuable opportunity for general practitioners and other health professionals to develop new screening tools which are accessible, portable, reliable and which can combine multiple tests within a single device. ${ }^{1}$ The measurement of visual acuity is one area that may benefit significantly from

\section{ARTICLE SUMMARY}

Article focus

- Do tests of visual acuity displayed on an iPad give results which are significantly different to those gained from a standard letter chart of the same design?

- Which environmental factors influence the results of tests displayed on an iPad?

Key messages

- Measurements of visual acuity using the iPad are highly susceptible to glare.

- When sources of glare are removed the iPad can provide measurements of distance visual acuity that are indistinguishable from measures using a standard letter chart.

Strengths and limitations of this study

- This study only investigated visual acuity measurement on an $\mathrm{iPad}$, and did not investigate other tablet devices with similar size screens.

the use of new technology. Visual acuity is a core diagnostic measure of visual function used across a broad range of healthcare fields including general practice. Acuity measurements are also made in a variety of other contexts such as school screening, driver licensing and occupational testing.

Despite the extensive use of visual acuity measurement, visual acuity is often not reported in referrals into ophthalmology services, ${ }^{2}$ possibly due to uncertainty relating to measurement conditions and reporting protocols for visual acuity. For example, accurate assessment of visual acuity critically depends on a range of factors such as viewing distance, chart illumination, the type of eye chart used and the scoring technique employed. It is also notable that the most commonly used method of measuring visual acuity, the Snellen chart, contains letters that are not equally legible and has unequal letter and line spacing that may result in inaccurate measurements. ${ }^{34}$ Accurate and reliable charts exist, ${ }^{5-7}$ but have not yet displaced the Snellen chart. 
Tablet computer technology has the potential to resolve these issues. Highly portable devices such as the Apple iPad and a range of similar products with relatively large, high resolution screens that have followed are able to display an assortment of letter and optotype (test symbol) charts at both high and low contrast. Importantly, these devices support software that can facilitate the choice of age appropriate standardised charts, randomise letters or optotypes to prevent chart learning, adjust stimulus size to accommodate the available viewing distance, vary the screen illumination depending on the lighting conditions and store or transmit the data collected. In addition, software running on such devices can ensure correct scoring of visual acuity. This is important as the choice of an appropriate scoring system for a specific combination of chart type and viewing distance is required for accurate measurements that are repeatable across multiple testing sites and testers. ${ }^{8}$

Before portable tablet devices can be used for acuity testing, however, it is necessary to ensure that the stimuli presented comply with international standards relating to letter size, spacing, contrast and luminance ${ }^{9}$ and that the measurements made using such devices are in agreement with those made using established systems. The aim of this study was to assess the suitability of portable tablet devices for visual acuity assessment in adults with no reported ocular pathology.

\section{METHODS}

Participants

Participants were recruited by convenience sampling whereby email lists and poster advertising at the University of Auckland were used to advertise the study. Fifty-six participants (average age 28, range 19-66) took part in the first set of measurements which used the iPad without an antiglare screen in place. Of the 56 participants, 26 were aided with spectacles and 29 were unaided. Twenty-nine participants (16 women, 13 men, average age 27.4, range 16-62) took part in the second, separate set of measurements for which an antiglare screen was fitted to the iPad. Of these 29 participants, 19 were aided with spectacles and the remaining 10 were habitually unaided. Only six participants took part in both sets of measurements. Exclusion criteria for both sets of measurements included visual acuity worse than 6/60 measured using the ETDRS chart, any selfreported history of ocular pathology and the use of contact lenses rather than spectacle correction. We note that self-report does not rule out all forms of ocular pathology. Participants were tested with test-distance-appropriate habitual refractive correction in place. The study was approved by the University of Auckland Human Ethics Committee; all participants provided full, written informed consent and all study protocols were in accordance with the Declaration of Helsinki.

\section{Apparatus and test environment}

Visual acuity was measured using a first generation Apple iPad, an ETDRS letter chart, an externally illuminated Bailey Lovie Letter Chart, an externally illuminated HOTV letter chart and a Medmont computerised visual acuity testing system (model AT20R, Vermont, Australia; figure 1) that presented a digital version of the Bailey-Lovie chart. The iPad (V.1, screen size 9.7", screen resolution $768 \times 1024$ ) was set to maximum brightness in the display settings throughout all testing procedures. For vision testing, the iPad was equipped with Visual Acuity XL, a commercially available visual acuity testing application sold by Kybervision (Montreal, Canada) through the iTunes store. This software was chosen as it is one of the few visual acuity measurement applications for the iPad that complies with recommendations made by the National Eye Institute and International Council of Ophthalmology. ${ }^{10}$ This software also allows for an iPod Touch to be used as a remote control/response device via a wireless connection with the iPad. A second generation 8 gigabyte iPod Touch was used for this purpose. After the first set of acuity measurements had been made, the iPad was fitted with an antiglare screen (Sentry Anti-Glare Screen Protector, Enki, Atlanta, Georgia, USA) and positioned away from sources of glare. The antiglare screen incorporates a matte surface which diffuses specular reflections and therefore reduces the detrimental effect of veiling glare on contrast. The Medmont software was run on a Dell desktop computer and displayed the visual stimuli via a liquid crystal display (LCD) computer monitor with a matte surface screen (14 inch, $1280 \times 1024)$, and a Bluetooth remote control device.

A full $6 \mathrm{~m}$ lane (charts were viewed directly not by reflection) was used to make all measurements. The test room was lit by fluorescent tubes (Philips TLD65) that gave a quoted colour temperature of $6500 \mathrm{~K}$ and had a colour-rendering index of 98 (to simulate daylight). A Minolta LM-100 photometer was used to measure the luminance of the optotypes and background for all testing systems.

\section{Procedure}

This study consisted of two sets of measurements. The first set was made using the iPad with no antiglare screen, the printed externally illuminated ETDRS chart and the Medmont system each displaying letter optotypes scaled and spaced in LogMAR units. The second, separate set of measurements was made using the iPad under conditions which minimised glare. These included positioning the iPad away from all glare sources and equipping the iPad with an antiglare screen. Comparisons were made between the iPad Sloan chart, the ETDRS chart, the Medmont system and a Bailey-Lovie chart. The second set of measurements also included the presentation of HOTV letters on the iPad in LogMAR progression and the use of a printed externally illuminated HOTV chart. The HOTV test was 


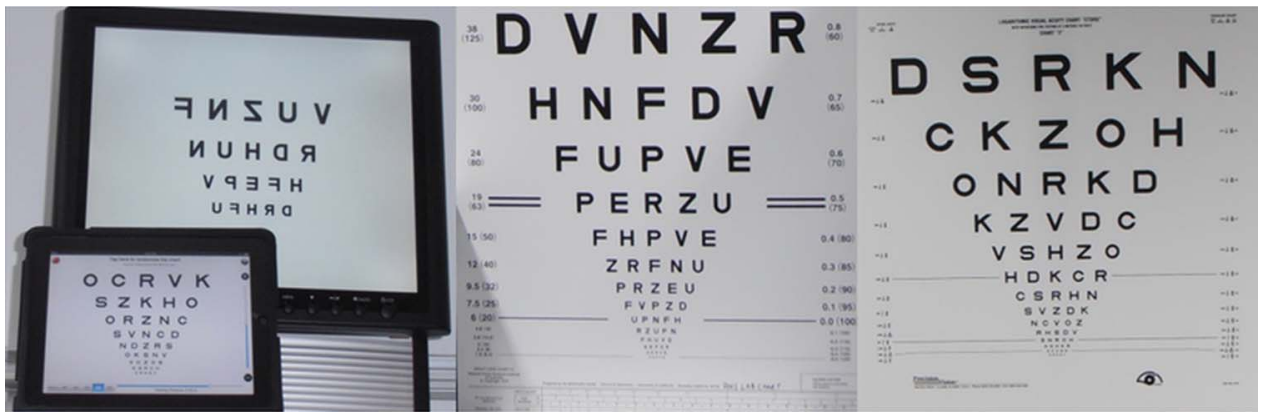

Figure 1 The visual acuity tests which were used for comparison. The Medmont computerised visual acuity system (top left), the iPad (bottom left), the Bailey Lovie Chart (middle) and the ETDRS letter chart (right).

included as it is commonly used for paediatric assessment. Measurements were first made with habitual spectacle correction in place and were then repeated with +1 dioptre of optical defocus added to the participants' habitual correction using a trial frame. The HOTV stimuli were not assessed under blurred conditions as our adult participants had a $25 \%$ chance of guessing the correct letter. The sequence of testing was randomised for the first set of measurements and the same test order was repeated for the measurements made with optical defocus.

For all measurements, the participants were tested by one of the two experienced visual acuity testers (final year optometry students). The starting examiner was randomised across participants and then the two examiners administered alternate tests throughout the testing procedure. Each examiner was masked to the acuities recorded by the other examiner during the testing session.

For each test, participants were asked to read the first letter or last letter of each line starting from 0.7 LogMAR until hesitation was observed or they were no longer able to resolve the optotype. They were then instructed to read all the lines starting one line above the point at which they were stopped until they were able to correctly read only 3 of 5 letters correctly. Results were recorded in LogMAR units ( $\log$ minutes of arc). Testing was always conducted in the standard clinical order of right eye, left eye and both eyes together (binocular viewing). For the iPad and Medmont systems, the optotypes were randomised line by line. The ETDRS, Bailey-Lovie and HOTV charts were 'reversible' with a different sequence of letters printed on each side of the substrate. The side of the chart used was alternated from one measurement to the next for each participant.

For the iPad measurements made without strategies to reduce glare (measurement set 1 ), the device was positioned on a flat surface and stood at an almost vertical angle within a commercially available iPad case that could be configured into a stand (iPad stand, http:// www.apple.com). For the measurements made with antiglare strategies in place (measurement set 2), an antiglare screen was fitted to the iPad and the device was positioned on an acuity chart stand so that it was exactly perpendicular to the floor and so that no sources of glare were visible by reflection. Our experimental design generated one acuity measurement per participant for each of the following factors: chart type, viewing condition (left eye vs right eye vs binocular viewing) and blur (present vs absent). A repeated measures analysis of variance (ANOVA) was therefore used to assess the main effects and interactions relating to these factors. Post hoc paired samples t tests were also employed. Degrees of freedom within the ANOVA were corrected for sphericity where necessary using the Huynh-Feldt correction. For the second set of measurements, the data from the paediatric HOTV tests were treated separately. Intraclass correlation coefficients were used to compare binocular measurements made using the iPad Sloan letters and those made using the ETDRS chart.

\section{RESULTS}

There was no systematic variation in the physical dimensions of equivalent-sized optotypes across printed charts, the iPad and the Medmont system and the luminance of the iPad screen did not vary with available battery charge (test range $100 \%$ charge to $5 \%$ charge, average luminance $275 \mathrm{~cd} / \mathrm{m}^{2}$ ). The Weber contrast of the dark optotypes presented on a light background was 0.95 for the internally illuminated iPad (measured with no sources of glare), 0.85 for the externally illuminated printed ETDRS chart and 0.98 for the internally illuminated Medmont system.

The first set of acuity measurements indicated that in the absence of procedures to reduce glare, the iPad provided acuity measurements that were significantly worse by an average of 0.18 LogMAR (9 letters) than all other tests under both normal $\left(\mathrm{F}_{1,55}=56.05, \mathrm{p} \leq 0.01\right)$ and blurred $\left(\mathrm{F}_{1,65}=69.86, \mathrm{p} \leq 0.01\right)$ testing conditions (figure $\left.2 \mathrm{~A}\right)$. The intraclass correlation coefficient for measurements made using the iPad and ETDRS chart under binocular viewing conditions was $0.40(95 \% \mathrm{CI}-0.13$ to 0.67$)$.

The second set of measurements was made with an antiglare screen attached to the iPad glass and with the device positioned to avoid reflections from any overhead glare sources. This antiglare screen did not reduce the luminance contrast of the optotypes presented on the 

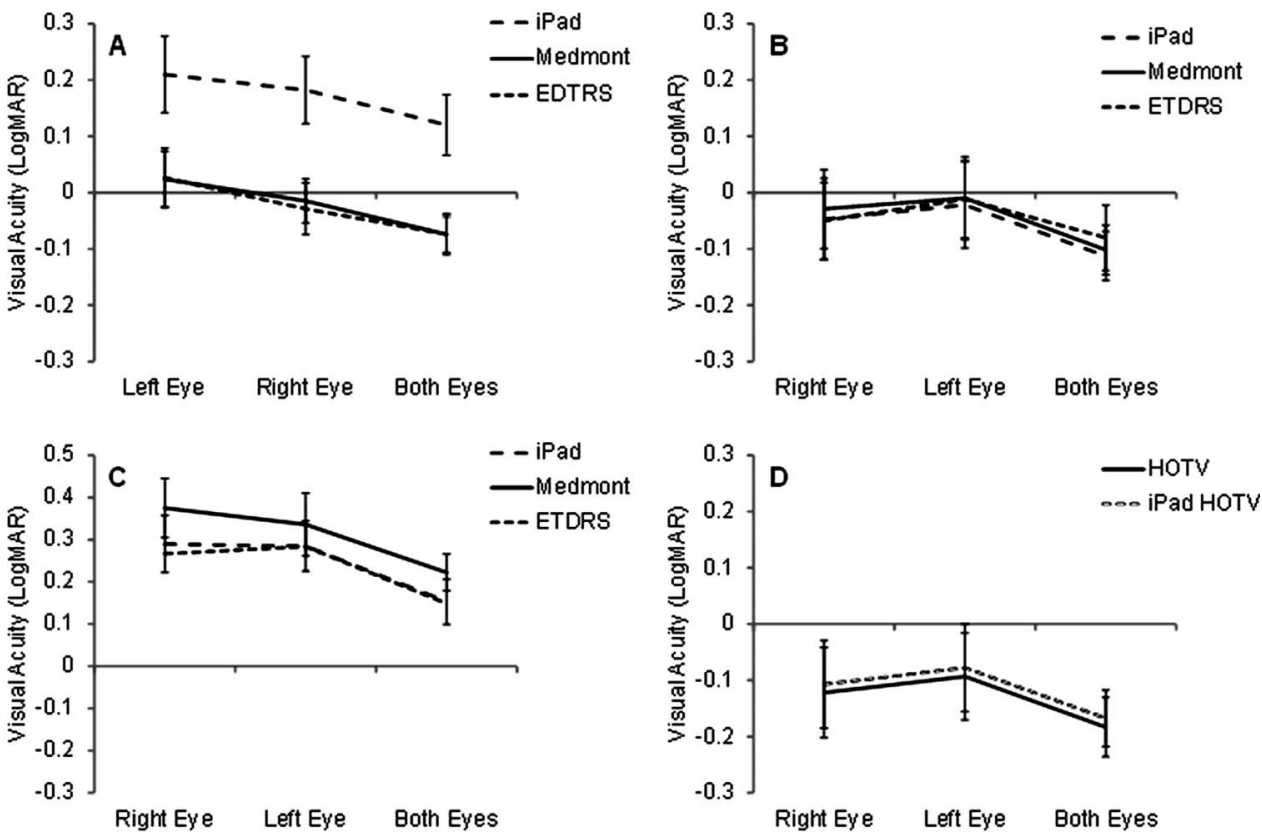

Figure 2 Visual acuity measurements made using the iPad, printed charts and the Medmont acuity testing system. (A) The effect of glare on the iPad which gave significantly worse acuity measurements than the comparison charts (measurement set $1, n=56$ ). (B) A separate set of measurements made when glare was minimised (measurement set 2, $n=29$ ). Under these conditions, the iPad measurements are accurate. (C) Measurements made with glare accounted for and optical defocus in place (measurement set 2, $n=29$ ). (D) Measurements made using the paediatric HOTV symbols shown on a printed chart and the iPad (measurement set $2, n=29$ ). The iPad provided accurate measurements when glare was minimised. Errors bars represent $95 \% \mathrm{Cls}$.

iPad (Weber contrast $=0.98$ ). ANOVA revealed a significant interaction between chart type and blur for the measurements made using standard letter optotypes $\left(\mathrm{F}_{2,63}=4.1, \mathrm{p}=0.02\right)$. In the absence of blur, there were no statistically significant differences between the charts (figure 2B; no main effect of chart type, $\mathrm{p}>0.05$ ). When blur was present the charts did differ from one another $\left(\mathrm{F}_{2,55}=4.9, \mathrm{p}=0.012\right.$, figure $\left.2 \mathrm{C}\right)$; however, the iPad did not differ from the gold standard ETDRS chart $(p>0.05)$. The intraclass correlation coefficient for measurements made using the iPad Sloan and ETDRS chart under binocular viewing conditions was 0.77 (95\% CI 0.52 to 0.90 ).

Acuity measurements made with HOTV letters were an average of $0.02 \mathrm{LogMAR}$ better ( 1 letter) when measured using the printed chart relative to the iPad (Figure 1D). This difference approached statistical significance $\left(\mathrm{F}_{28}=3.8, \mathrm{p}=0.06\right)$, but is not likely to be clinically significant. ${ }^{11} 12$

\section{DISCUSSION}

Two main conclusions can be drawn from the results of this study. First, acuity measurements made using the iPad device are highly susceptible to glare. Second, when sources of glare are minimised, the iPad device can provide measures of distance visual acuity that are indistinguishable from measures made using gold standard letter charts. The implication of these findings is that while the iPad can be used to present acuity charts in situations where sources of glare can be properly controlled, the system is not suitable for testing in uncontrolled environments. To emphasise this point, we found that even with an antiglare screen in place, it was still necessary to orient the iPad exactly perpendicular to the floor and position the device away from overhead lighting sources to prevent the contrast of the display being detrimentally affected by glare.

Therefore, in situations where sources of glare can be controlled, the iPad represents a relatively inexpensive, flexible testing platform which can be updated and changed as necessary via a simple software revision. In addition, as vision testing is often conducted in remote locations in conjunction with outreach programmes offering cataract surgery and the treatment of eye disease; combining multiple tests into one device is highly desirable. Charts presented using the iPad platform can also be operated by a handheld remote that allows for optotype matching and software can provide instructions in multiple languages and support accurate scoring of behavioural results. Furthermore, the iPad system allows for the randomisation of letters which facilitates accurate retesting by minimising chart learning. Finally, the iPad has the advantage that other health-related software could be loaded onto the device and data could be transmitted wirelessly to a central database. Our results are consistent with a previous report demonstrating that tumbling $\mathrm{E}$ letters presented on an iPad device can provide accurate measures of visual acuity for observers with acuities better than $6 / 60 .^{13}$ Interestingly, iPad measurements became inaccurate 
relative to a conventional internally illuminated tumbling E chart for observers with acuities worse the 6/60 in this study.

Although this study focused on the iPad, it is likely that our results apply to a range of tablet devices with similar size screens. Also, since the initiation of this study, new generations of iPad have been released that have higher resolution screens. Our results are still relevant; however, as we found that distance acuity measurements conducted at $6 \mathrm{~m}$ were not limited by the resolution of the screen, as they were indistinguishable from the printed ETDRS chart measurements. However, it is conceivable that these new systems may allow for the accurate measurement of near visual acuity.

Contributors JB, RJ, GP and BT were involved in design of the study, monitoring of data collection, statistical analysis and manuscript preparation. LC, AT and ET recruited participants, collected and analysed data and were involved in editing of the final manuscript.

Funding This research received no specific grant from any funding agency in the public, commercial or not-for-profit sectors.

Competing interests None.

Ethics approval University of Auckland Human Participants Ethics Committee.

Provenance and peer review Not commissioned; externally peer reviewed.

Data sharing statement No additional data are available.

\section{REFERENCES}

1. Berger E. The iPad: gadget or medical godsend? Ann Emerg Med 2010;56:A21-2.

2. Rao GP, Moriarty AP. Measuring visual acuity in general practice. Many referral letters omit visual acuity measurement. BMJ 1995;310:671.

3. McGraw P, Winn B, Whitaker D. Reliability of the Snellen chart. BMJ 1995;310:1481-2

4. Currie Z, Bhan A, Pepper I. Reliability of Snellen charts for testing visual acuity for driving: prospective study and postal questionnaire. BMJ 2000;321:990-2.

5. Bourne RR, Rosser DA, Sukudom P, et al. Evaluating a new logMAR chart designed to improve visual acuity assessment in population-based surveys. Eye (London) 2003;17:754-8.

6. Bailey IL, Lovie JE. New design principles for visual acuity letter charts. Am J Optom Physiol Opt 1976;53:740-5.

7. Ferris FL III, Kassoff A, Bresnick GH, et al. New visual acuity charts for clinical research. Am J Ophthalmol 1982;94:91-6.

8. Dong LM, Marsh MJ, Hawkins BS. Measurement and analysis of visual acuity in multicenter randomized clinical trials in the United States: findings from a survey. Ophthalmic Epidemiol 2003;10:149-65.

9. BS-4274-1: Visual Acuity test types. Part 1: test charts for clinical determination of distance visual acuity—-specification: BSi, 2003:9.

10. (ICOPH) ICoO. Visual Acuity Measurement Standard. Visual Functions Committee. Ital J Ophthalmol, 1984.

11. Arditi A, Cagenello R. On the statistical reliability of letter-chart visual acuity measurements. Invest Ophthalmol Vis Sci 1993;34: $120-9$.

12. Shah N, Laidlaw DA, Shah SP, et al. Computerized repeating and averaging improve the test-retest variability of ETDRS visual acuity measurements: implications for sensitivity and specificity. Invest Ophthalmol Vis Sci 2011;52:9397-402.

13. Zhang ZT, Zhang SC, Huang XG, et al. A pilot trial of the iPad tablet computer as a portable device for visual acuity testing. J Telemed Telecare 2013;19:55-9. 